\title{
Ultrastructural analysis of fine needle aspirates from benign breast lesions
}

\author{
D J P Ferguson, C A Wells, V Crucioli ${ }^{\star}$
}

\begin{abstract}
Thirty six fine needle aspirates from various types of benign breast lesions were examined by electron microscopy and correlated with their cytological appearances. In all cases the parenchyma consisted of clumps of cohesive cells with the ultrastructural features of epithelial cells. In many cases, particularly from fibroadenomas, the parenchyma consisted of single layers of polarised epithelial cells showing lumen formation. Similar arrays of apocrine epithelial cells were observed in $60 \%$ of fibrocystic lesions. The more solid clumps from hyperplastic lesions consisted of epithelial cells of variable shape and electron density with disorganised lumen formation. Irrespective of the type of lesion, the epithelial cells were not normally subtended by myoepithelial cells or basal lamina. The extraction process seems to result in a shearing between the epithelium and basal lamina with lysis of the myoepithelial cells. Most naked nuclei probably result from lysed myoepithelial cells
\end{abstract}

Fine needle aspiration cytology is now widely used in the diagnosis of breast disease. It provides a rapid and accurate result and is relatively non-invasive. In benign diagnosis it can eliminate the need for surgical biopsy with an associated saving in resources. The procedure entails the removal of a cell sample through a fine needle by applying suction. Cytological features can accurately differentiate benign from malignant lesions. ${ }^{1}$ In the case of benign lesions, this means the cell size, cell regularity, and the identification of two populations, characterised as epithelial and myoepithelial cells on the basis of nuclear morphology. These features can also be used to differentiate between types of benign lesions. ${ }^{1}$ The effect of the extraction process on the characteristic in situ architecture of the breast parenchyma is not clearly understood, however. In this study we correlated the ultrastructural appearances with cytological features in an attempt to understand the effect of aspiration on the architecture and cells of the parenchyma in benign breast lesions and compared the appearances with the morphology in excision biopsy specimens.

Methods

Fine needle aspiration cytology was carried out on breast lumps of women attending the Breast Clinic at this hospital. The aspirates were used to prepare smears for cytological diagnosis in the normal manner. Subsequently any remaining material was expelled into $4 \%$ glutaraldehyde in phosphate buffer and processed for electron microscopy. The samples were postfixed in osmium tetroxide, dehydrated, and embedded in epoxy resin (Emix). Sections (1 $\mu \mathrm{m}$ ) stained with Azure A were used to identify parenchymal components by light microscopy. Thin sections of suitable areas were stained with uranyl acetate and lead citrate before electron microscopic examination. This study was based on 36 samples identified as comprising benign epithelium by electron microscopy, without knowledge of the cytological diagnosis.

\section{Results}

The 36 benign aspirates identified by their ultrastructural appearances correlated with 31 benign, three suspicious (two benign and one malignant by histology), and two malignant aspirates diagnosed by routine cytology (table). In the cases diagnosed as malignant both benign and malignant components were observed by cytology, although only benign epithelium was present in the portion processed for electron microscopy. The cytological characteristics used to diagnose and differentiate between benign lesions were as described by others (fig 1). ${ }^{1}$ The incidence of the various types of lesions in this study is given in the table.

Ultrastructurally, the parenchyma from all benign lesions consisted of cohesive clumps of epithelial cells. Single intact epithelial cells were not observed. The nucleus and cytoplasmic organelles were similar to those described for epithelial cells in excision biopsy specimens. ${ }^{23}$ In most cases, irrespective of the type of lesion characterised by cytology, a distinctive architecture was seen. The cell clumps consisted of a single layer of polarised epithelial cells forming complete or partial

Cytological diagnosis of cases identified as having benign epithelium on electron microscopy

\begin{tabular}{lc}
\hline Cytology & Number \\
\hline Malignant & 2 \\
Suspicious & 3 \\
Fibrocystic change` & 15 \\
Fibroadenoma & 10 \\
Juvenile papillomatosis & 1 \\
Benign (not otherwise specified) & 5 \\
Total & 36
\end{tabular}

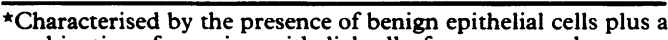
combination of apocrine epithelial cells, foamy macrophages, or
cyst debris. 
Figure 1 Light micrograph of the cytological appearance of a cell clump from $a$ benign aspirate. Note two types.of epithelial cells

Figure 3 Electron micrograph of a benign aspirate showing $a$ ductule lined by a single layer of epithelial cells (E). Note absence of underlying myoepithelia cells and basal lamina. Lu-lumen.

Insert Detail from apex of two epithelial cells showing lumen formation consisting of microvilli (Mv) and the junctional complex of tight ( $T$ ) and intermediate (I) junctions plus desmosomes (D).

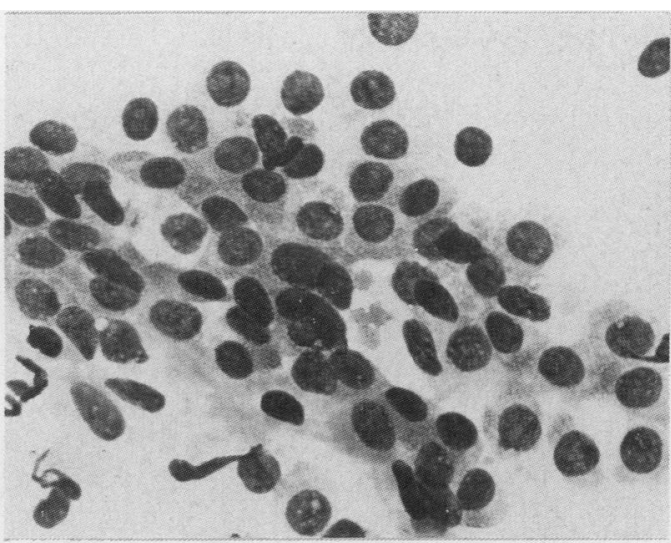

ducts or acini showing lumen formation. Such appearances could be identified by both light and electron microscopy (figs 2 and 3). Ultrastructurally, lumen formation was identified by apical junctional complexes and the presence of microvilli (fig 3, insert). Extensive areas of such parenchyma were observed in cases characterised as fibroadenomas by cytology. Sixty per cent of the cases diagnosed as fibrocystic disease also contained similarly organised apocrine epithelial cells (fig 4). These were identified by their large size with numerous electron dense vacuoles in the apical cytoplasm and extensive folding of lateral membranes. In either case the parenchyma consisted of a single layer of epithelial cells with no evidence of underlying myoepithelial cells or basal lamina. Examination of the basal region of the cells showed cell remnants attached by desmosomes to the epithelial cells (fig 4, insert) and occasionally the nucleus of a lysed basal cell was present (fig 2).

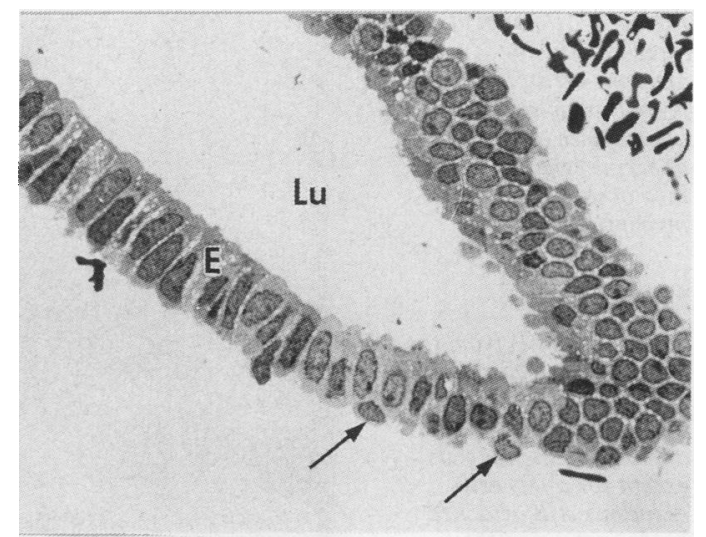

Figure 2 Light micrograph of a section through an aspirate processed for electron microscopy. Note portion of duct lined by epithelial cells $(E)$ but lacking myoepithelial cells except for a few nuclei (arrows). Lulumen.

In cases with epithelial hyperplasia solid clumps of epithelial cells were present (fig 5, insert). Many of these clumps comprised epithelial cells exhibiting considerable variation in shape and electron density. The cells varied from cuboid to spindle shaped, forming a disorganised multilayered structure with multiple small areas of lumen formation (fig 5). The cells, irrespective of shape, showed the ultrastructural features of epithelial cells with no evidence of the smooth muscle differentiation characteristic of myoepithelial cells. In certain examples long spindle shaped cells showed evidence of lumen formation (fig 5). A few lymphocytes/monocytes, identified by their nuclear and cytoplasmic morphology, were present between the epithelial cells. In

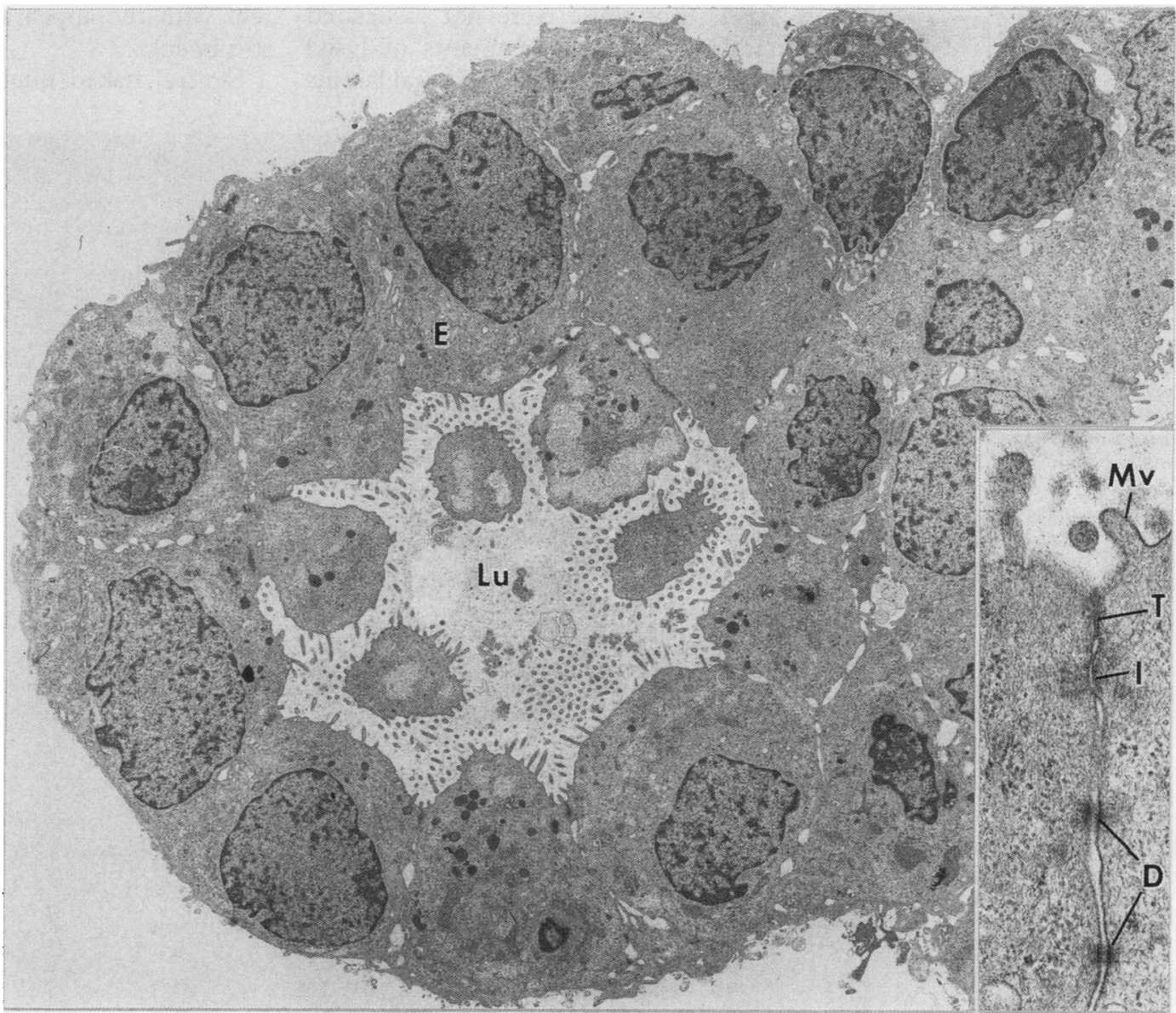


Figure 4 Portion of an aspirate showing apocrine metaplasia. Note characteristic appearance of the apocrine cells but the lack of underlying myoepithelial cells.

Insert Details from the basal region of the epithelial cells showing the lack of basal lamina and myoepithelial cells except for a few cell remnants still attached by desmosomes $(D)$.

Figure 5 Part of $a$ clump of hyperplastic epithelial cells illustrating the variation in shape and electron density of the cells with multiple areas of lumen formation. Note dense elongated cell characterised as epithelial by lumen formation (arrow). Lu-lumen, $\mathrm{Ma}$ macrophage.

Insert Light micrograph of cell clump showing the variability in appearance of the epithelial cells.

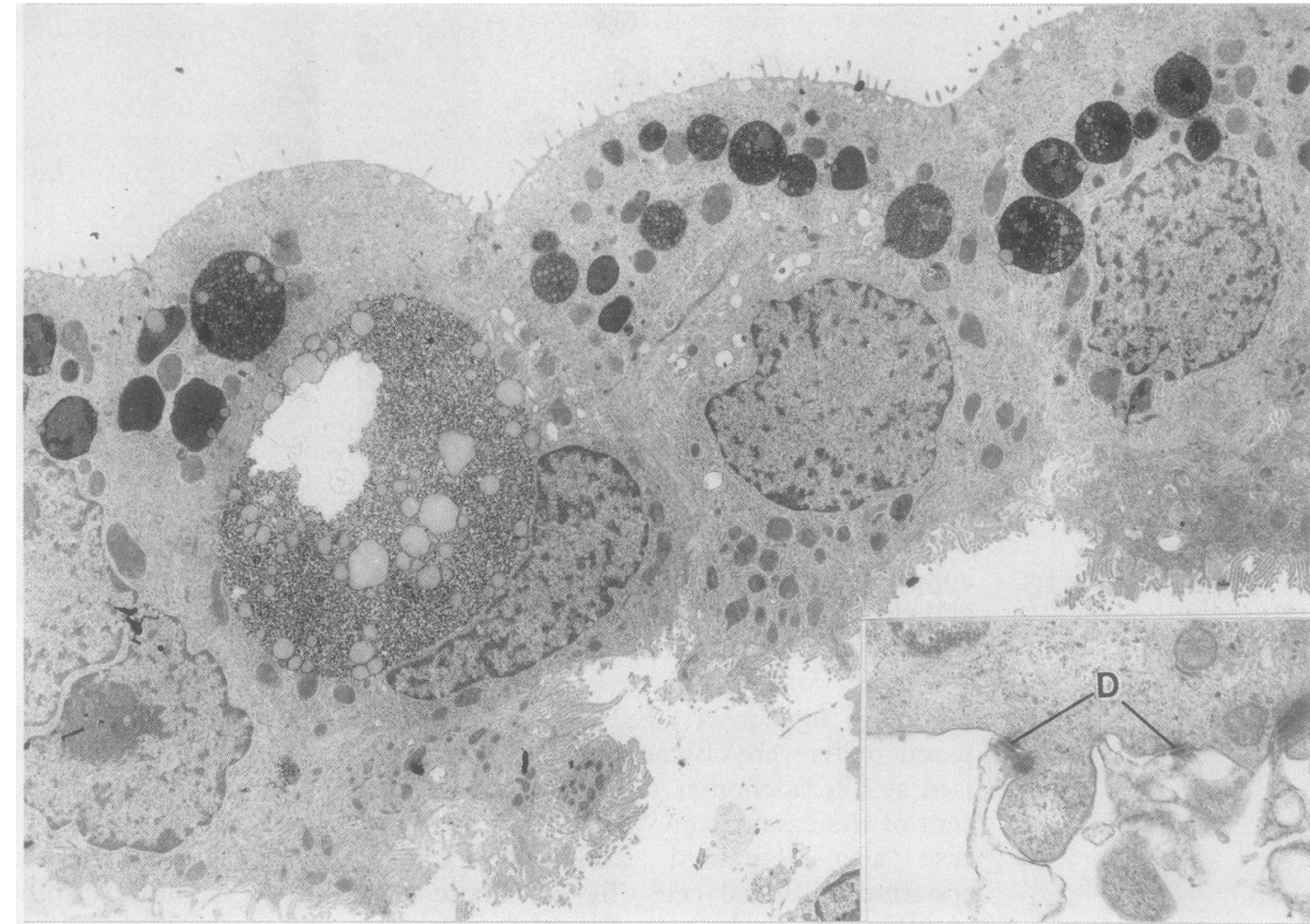

other examples the epithelial cells were more uniform in staining and shape (fig 6). These cells differed from the cells in normal epithelium in that they were slightly larger with a proportion of the cells having large irregular nuclei and nucleoli. Although lumen formation was observed, there were multilayers of these cells with no luminal differentiation.

After careful examination we observed very occasional areas of basal lamina and connective tissue (fig 7), but these were not associated with intact parenchyma. Remnants of lysed myoepithelial cells attached to the basal lamina

by hemidesmosomes were identified (fig 7).

In contrast, tissue fragments were seen only rarely: only two fragments were observed in the 36 aspirates examined. These differed from the normal aspirated parenchyma in retaining the in situ architectural organisation (fig 8)-that is, acini composed of central epithelial cells with underlying myoepithelial cells, surrounded by a basal lamina, and embedded in connective tissue (fig 8 , insert). This is consistent with the appearances in excision biopsy specimens.

Several naked nuclei were observed in all

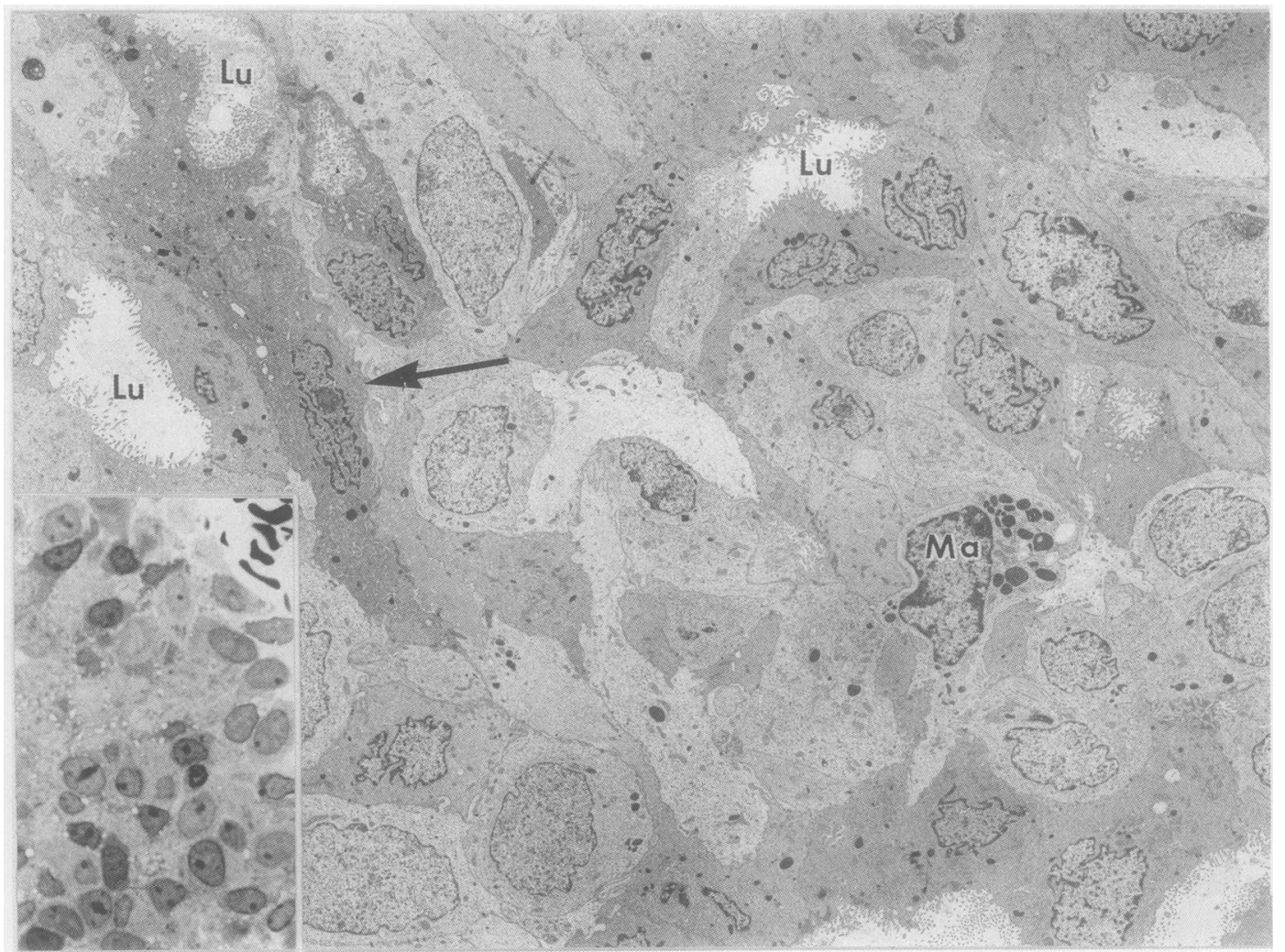


Figure 6 Electron micrograph of a clump of hyperplasic epithelial cells with a more monomorphic appearance and no evidence of lumen formation.
Figure 7 Electron micrograph illustrating a portion of basal lamina with underlying connective tissue. Note absence of overlying parenchymal cells except for remnants of the myoepthithelial cells still attached to the basal lamina. C-collagen, $H$-hemidesmosomes.

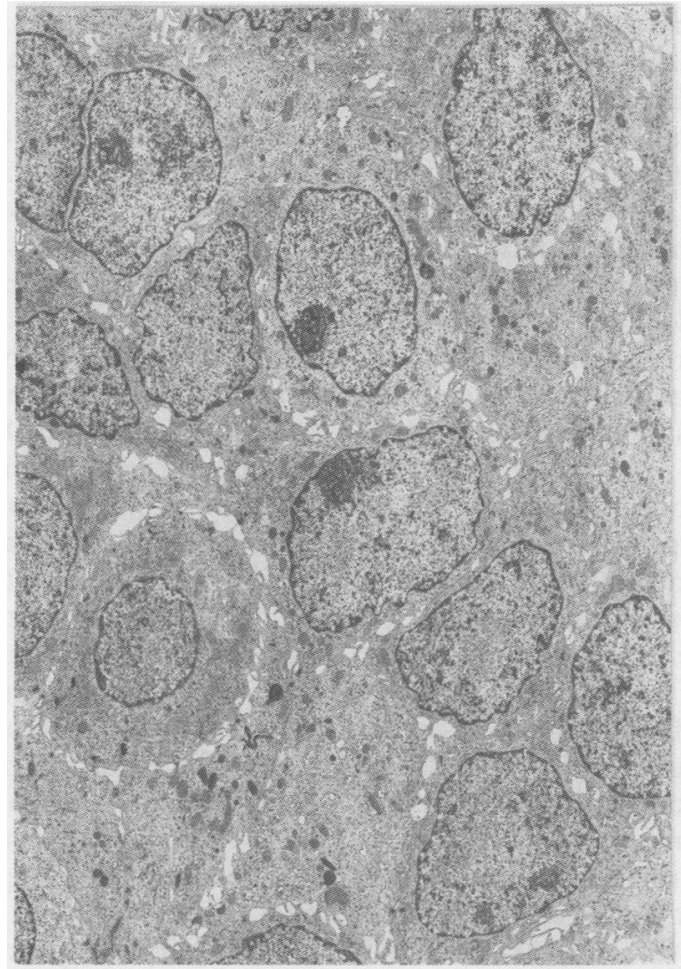

aspirates. These had a fine granular nucleoplasm with intact nuclear membranes. Absence of cytoplasm, however, prevented further characterisation of the cell of origin, although they were similar to those associated with lysed cells around the epithelial clumps-that is, myoepithelial cells. Lipid containing (fat) cells were also present in most aspirates.

A further characteristic of the aspirates from fibrocystic lesions was the presence of variable numbers of foamy macrophages. These were characterised as large cells with an eccentric nucleus and cytoplasm packed with numerous large vacuoles containing lipid and degenerate material.

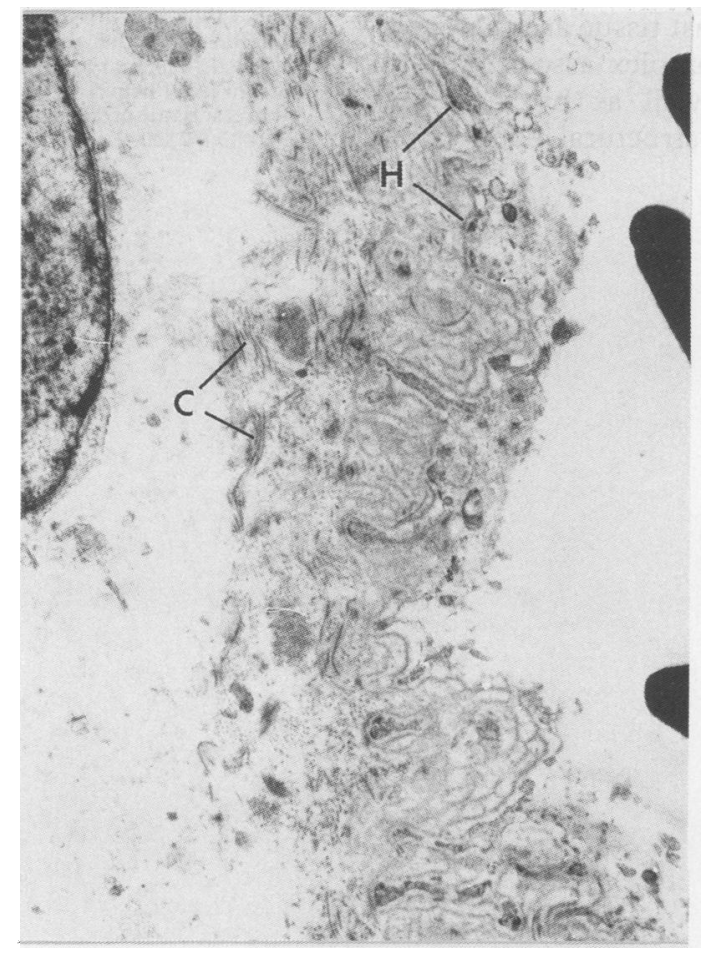

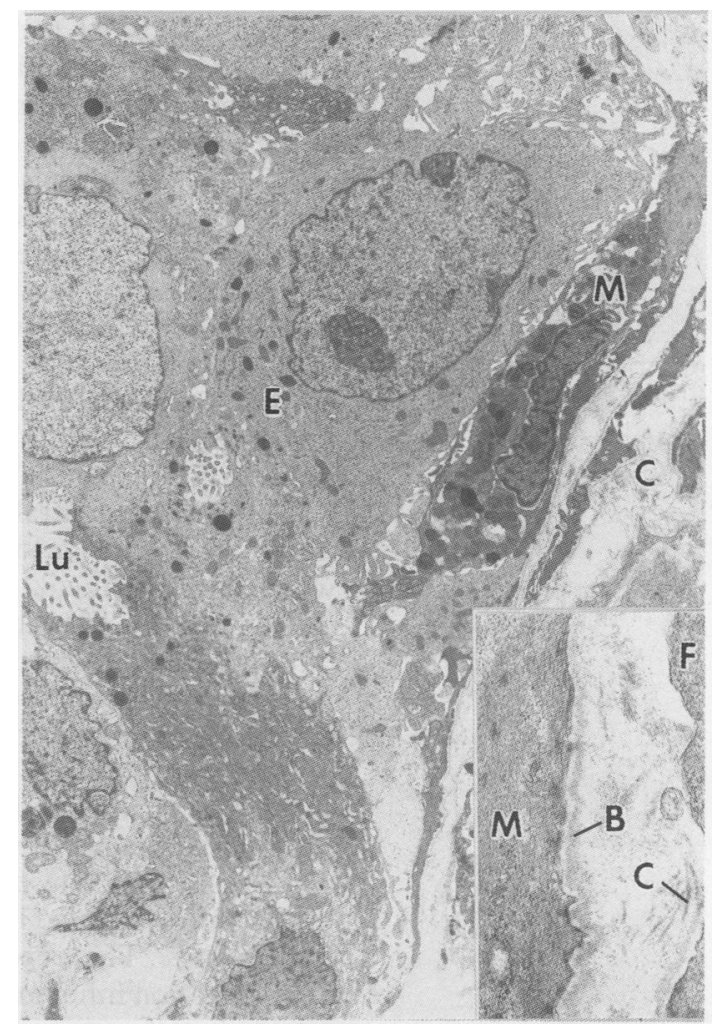

Figure 8 Part of an acinus from a rare example in which a portion of a lobule has been extracted intact showing the typical in situ relation of the parenchyma with epithelial $(E)$ and underlying myoepthelial $(M)$ cells surrounded by connective tissue (C). Lu-lumen.

Insert Details of periphery of acinus showing part of a myoepithelial cell $(M)$, basal lamina $(B)$, collagen fibre $(C)$ and fibroblast $(F)$.

\section{Discussion}

The 36 aspirates with benign parenchyma described in this study were identified from over 200 aspirates examined by electron microscopy. From the examination of 200 aspirates, in which all the extracted material was concentrated by centrifugation, it became clear that certain components of the breast are easier to extract and thus more commonly found than others. Red blood corpuscles and intact fat cells, for example, are most easily extracted followed by the parenchyma, either benign or malignant depending on the lesion, and lastly the connective tissue, which is rarely extracted, probably due to the interlocking nature of the collagen matrix. It is this abilty to remove preferentially the parenchyma which allows aspiration cytology to be a useful diagnostic technique.

The extraction process seems to disturb the normal in situ architecture of benign lesions. The clumps of enithelial cells were not subtended by intact myoepithelial cells or basal lamina which are characteristic features of benign lesions seen in excision biopsy specimens. ${ }^{4}$ This study emphasises that aspiration is not a form of microbiopsy because the finding of tissue fragments, which have the typical in situ organisation, is extremely rare.

The basal lamina, which separates the epithelial components from the connective tissues, seems to be firmly attached to the extracellular matrix and thus not easily extracted. The myoepithelial cells are also more firmly atta- 
ched to the basal lamina than the epithelial cells. This is probably due to the hemidesmosomes present along the basal plasmalemma of the myoepithelial cells. ${ }^{4}$ The myoepithelial cells, however, are also firmly attached to the adjacent epithelial cells by desmosomes and gap junctions. ${ }^{5}$ The extraction of the parenchyma is associated with a shearing of the parenchyma at the level of the myoepithelial cell/basal lamina. This results in the removal of intact epithelial cells and the rupture of the myoepithelial cell plasmalemma and subsequent lysis.

The ultrastructural observations seem to differ from the cytological descriptions in which both epithelial and myoepithelial cells are identified. ${ }^{1}$ This apparent anomaly may be due to differences in the processing technique combined with the fact that cytological identification of the myoepithelial cells is based on nuclear morphology alone. With the direct smearing process used for cytology, the nuclei of the lysed myoepithelial cells may retain their association with the epithelial cells, and such nuclei were observed in a few cases by electron microscopy. The repeated resuspension and centrifugation inherent in electron microscopic processing, however, probably result in the detatchment of many of the nuclei from the lysed cells.

Much of the morphological variation in benign hyperplastic lesions, which had been reported as representing epithelial and myoepithelial cells by cytology, seems to be the result of variations in the shape and electron density of epithelial cells with the elongated cells lacking smooth muscle differentation. This does not, however, detract from its usefulness in the cytological characterisation of benign aspirates.

The cohesive nature of the clumps of benign epithelial cells compared with clumps of malignant cells was observed by both cytology and electron microscopy. The epithelial cells in normal and benign breast tissue are characterised by a junctional complex associated with lumen formation, as well as by numerous desmosomes. $^{23}$ These structural connections probably explain the absence of single intact epithelial cells in benign aspirates, while they are a common finding in aspirates from most malignant lesions. This probably reflects the reduced structural differentiation, particularly with respect to lumen formation, associated with malignant transformation. This is with the exception of tubular carcinoma. In the aspirates from cases of tubular carcinoma, which we examined by electron microscopy, a similar architectural organisation was observed to that described for benign lesions - that is, a single layer of epithelial cells exhibiting lumen formation but no underlying myoepithelial cells or basal lamina (Ferguson and Wells, unpublished observations). They could often be differentiated on the basis of cell size and nuclear shape but may be more frequently diagnosed as suspicious due to the similarity of the ultrastructural features.

The fact that the benign parenchymal cells are firmly anchored to each other will mean that their removal will result in the rupture of the plasmalemma of the epithelial cell or myoepithelial cell at the point of extraction. Cell lysis resulting from rupture of the plasmalemma is probably responsible for the higher proportion of naked nuclei present in benign compared with malignant aspirates. Because the connective tissue is rarely extracted, it is unlikely that fibroblast nuclei contribute greatly to the number of naked nuclei observed.

Grateful thanks are given to Dr Crucioli without whose initiative this work would not have been possible. The cooperation of $M$ this work would not have been possible. The cooperation of $\mathrm{Mr}$ assistance of Mrs R Rychetnik and Mr A Skinner are gratefully acknowledged.

1 Oertel YC. Fine needle aspiration of the breast. Boston Butterworths, 1987

2 Ozzello L. Ultrastructure of the human mammary gland. Pathol Annu 1971;6:1-59.

3 Ferguson DJP. An ultrastructural study of mitosis and cytokinesis in normal "resting" human breast. Cell Tissue cytokinesis in normal "

4 Ozzello L. Epithelial-stromal junction of normal and dysplastic mammary glands. Cancer 1970;25:586-600

5 Strum JM, Phelps PC, McAtee MM. Resting human female breast tissue produces iodinated protein. J Ultrastruct Re 1983;84:130-9. 